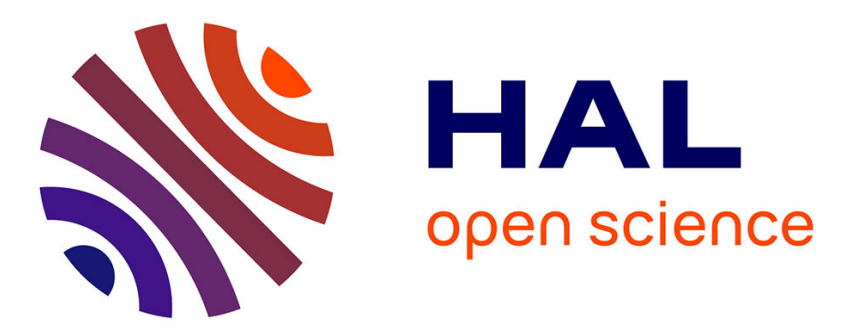

\title{
Structure haute fréquence et système de focalisation de l'accélérateur linéaire à ions lourds d'Orsay
}

\author{
C. Bieth, Ch. Goldstein
}

\section{To cite this version:}

C. Bieth, Ch. Goldstein. Structure haute fréquence et système de focalisation de l'accélérateur linéaire à ions lourds d'Orsay. Revue de Physique Appliquée, 1969, 4 (2), pp.181-182. 10.1051/rphysap:0196900402018100 . jpa-00243207

\section{HAL Id: jpa-00243207 https://hal.science/jpa-00243207}

Submitted on 1 Jan 1969

HAL is a multi-disciplinary open access archive for the deposit and dissemination of scientific research documents, whether they are published or not. The documents may come from teaching and research institutions in France or abroad, or from public or private research centers.
L'archive ouverte pluridisciplinaire HAL, est destinée au dépôt et à la diffusion de documents scientifiques de niveau recherche, publiés ou non, émanant des établissements d'enseignement et de recherche français ou étrangers, des laboratoires publics ou privés. 


\title{
STRUGTURE HAUTE FRÉQUENGE ET SYSTÈME DE FOGALISATION DE L'AGCÉLÉRATEUR LINÉAIRE A IONS LOURDS D'ORSAY
}

\author{
C. BIETH et Gh. GOLDSTEIN, \\ Institut de Physique Nucléaire, 91-Orsay.
}

\begin{abstract}
Résumé. - La cavité HF et le système de focalisation de l'accélérateur linéaire à ions lourds, qui est actuellement en construction à Orsay, sont brièvement décrits. Quelques résultats des mesures HF et de l'efficacité des grilles sont donnés.
\end{abstract}

Abstract. - The radiofrequency cavity and the focussing system for the heavy ion linac which is being constructed at Orsay, are briefly described. Some results on RF measurements and efficiency of the grids are given.

I. Structure haute fréquence. - La volonté d'obtenir un faisceau continu pour tous les ions que les sources actuelles ou futures sont ou seront capables de fournir elles-mêmes en continu, le choix du rapport $Z_{\mathrm{i}} / A \geqslant 0,1$, l'énergie finale de $1 \mathrm{MeV} /$ nucléon [1], ont entraîné les considérations suivantes : 1) nécessité d'améliorer l'impédance shunt effective des structures existantes en vue de réduire la puissance $\mathrm{HF}$ à fournir à la cavité et la puissance $\mathrm{HF}$ à évacuer par refroidissement dans l'ensemble de la structure; 2) nécessité de réduire la fréquence de travail en dessous de $30 \mathrm{MHz}$, afin de trouver les amplificateurs continus pouvant délivrer quelques centaines de $\mathrm{kW}$.

En 1962, au début de nos études, la structure qui pouvait paraître, en regard des considérations précédentes, la mieux adaptée, restait celle de Wideroe. Toutefois, la structure réalisée à Manchester présentait une impédance shunt médiocre et pour une accélération totale de $8 \mathrm{MV}$, la puissance HF nécessaire atteignait $400 \mathrm{~kW}$ à $25 \mathrm{MHz}$ [2].

Nous avons donc recherché l'ensemble des amélio-

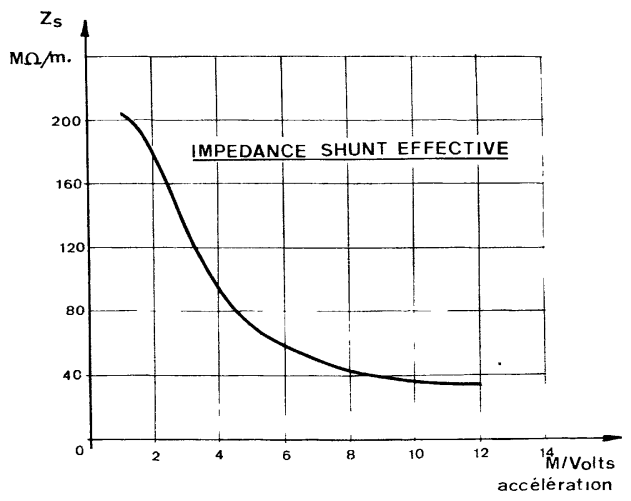

FIG. 1. rations possibles sur cette structure. En effectuant les calculs par la même méthode que celle utilisée pour les cavités de cyclotron [3], nous avons pu déterminer simultanément la distribution de tension, de courant, la puissance dissipée dans chaque élément, la fréquence de résonance en fonction de la position des courtscircuits [4]. La courbe de la figure 1 montre l'évolution de l'impédance shunt effective en fonction de la tension d'accélération pour $Z_{\mathrm{i}} / A \geqslant 0,1$ et $f=25 \mathrm{MHz}$.

Les mesures sur la cavité, dont CSF a achevé la construction en janvier 1968, ont donné les résultats suivants : i) fréquence de résonance : $25 \mathrm{MHz}$ (calculée), 24,4 (mesurée); ii) coefficient de surtension : 6000 (calculée), 5800 (mesurée); iii) distribution de tension ( fig. 2).

Ceci conduit en définitive, pour $Z_{\mathrm{i}} / A \geqslant 0,1$ et pour une accélération totale de $11,5 \mathrm{MV}$ par charge, à une puissance $\mathrm{HF}$ de $240 \mathrm{~kW}$, soit une impédance shunt effective moyenne : $Z_{\mathrm{s}}=\frac{\left(E_{\text {finale }}-E_{\text {initiale }}\right)^{2}}{P L}$ de $60 \mathrm{M} \Omega / \mathrm{m}$.

Notons à titre indicatif que la puissance nécessaire à Berkeley et à Manchester, pour la même accélération totale, serait de l'ordre de $800 \mathrm{~kW}$.

Les essais en puissance sont actuellement en cours et l'on a atteint une puissance de $35 \mathrm{~kW}$ soit, à l'extrémité de la ligne, une tension d'environ $200 \mathrm{kV}$. Toutefois, divers problèmes technologiques restent à résoudre sur la boucle de couplage (refroidissement, agrandissement de la surface de couplage, compensation du terme réactif) qui retardent actuellement la montée en puissance.

II. Electrodes d'accélération. - Notre calcul de la forme et de la longueur des électrodes est basé sur les critères de claquage de Kilpatrick; les résultats d'Heidelberg montrent combien ce problème est mal connu 
puisque des champs bien supérieurs à ceux prédits par Kilpatrick peuvent être utilisés. D'autre part, l'accélérateur de Manchester obtient $Z_{\mathrm{i}} / A \geqslant 0,1$ (au lieu de 0,125$)$. Nous pensons donc que, sous réserve d'un vide propre, il nous sera possible de diminuer $Z_{\mathrm{i}} / A$ en dessous de 0,1 , ce qui est favorable à l'accélération d'ions plus lourds ou à l'obtention d'une plus grande intensité.

Le nombre et la longueur des électrodes modifiant la distribution de tension, il est nécessaire d'effectuer plusieurs calculs des longueurs et d'atteindre une convergence entre le calcul des longueurs et la tension effectivement mesurée. G'est ainsi que, sur la maquette à échelle $1 / 4$, trois jeux consécutifs d'électrodes ont été

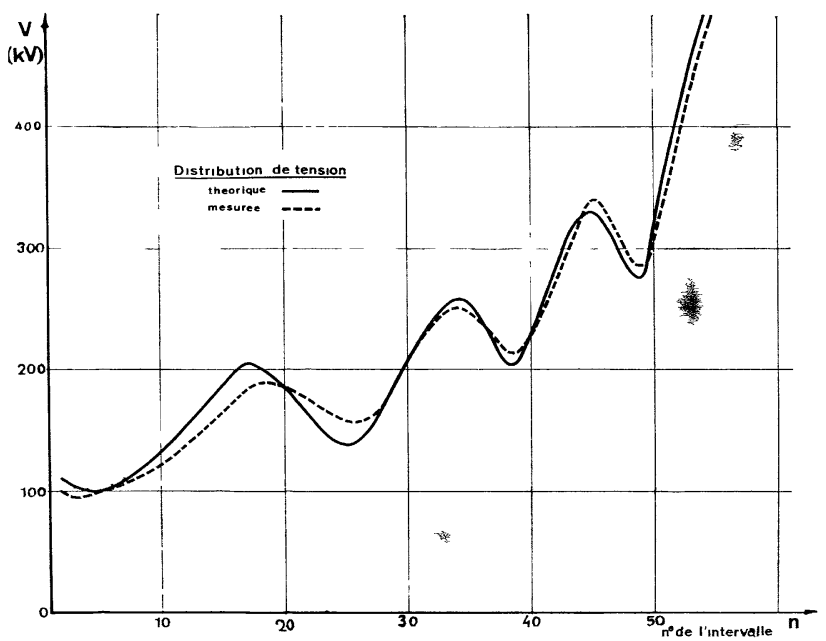

FIG. 2. réalisés. Le nombre total d'électrodes est finalement de 54 .

Les mesures de distribution de tension ont été effectuées par la méthode des perturbations en utilisant une bille de $8 \mathrm{~mm}$ de diamètre. Les résultats qui apparaissent sur la figure 2 conduisent à des oscillations de phases [5]. Toutefois, l'acceptance en phase reste voisine de $60^{\circ}$ et, sous réserve d'un contrôle de l'acceptance radiale, cette distribution semble convenir pour un premier essai de faisceau, prévu au début de 1969.

III. Système de focalisation. - Le choix d'un système de focalisation est fixé essentiellement par le mode d'accélération, la fréquence $\mathrm{HF}$, la vitesse d'injection et par les considérations technologiques qui en découlent : possibilité d'atteindre des champs magnétiques élevés dans les quadrupôles, de les loger dans les électrodes, de les refroidir et de les alimenter surtout lorsque le faisceau est continu. L'examen de ces impératifs nous a rapidement conduits à utiliser, comme à Berkeley et Manchester, la focalisation par grille. Cependant, à la lumière des études sur la focalisation par le champ HF lui-même [6], il nous a semblé possible, en utilisant des grilles plates croisées, d'obtenir à la fois une meilleure transparence et une acceptance radiale accrue. Les essais sur une maquette de vingttrois électrodes, d'un faisceau d'hélium $\left(Z_{\mathrm{i}} / A=0,25\right)$ accéléré à $200 \mathrm{keV}$, ont donné un rendement de $14 \%$ [7]. On peut rapprocher ce résultat de celui obtenu sur le même modèle avec une structure à doigts et qui atteint $20 \%$ [8]. Le rendement total de l'accélérateur devrait donc être, sans buncher, de 8 à $10 \%$, et avec buncher à harmoniques [9], de 30 à $40 \%$.

\section{BIBLIOGRAPHIE}

[1] Cabrespine (A.), ce colloque.

[2] Nassibian (G.), Rev. Sci. Instr., 1961, 32, 1316.

[3] Cabrespine (A.), Dupuis (Y.) et Bieth (C.), C.E.R.N. 63-19, 1963, 256.

[4] Bosser (J.), Thèse C.N.A.M., 1966.

[5] GoldDSTEIN (Ch.), Rapport interne 1968, à paraître.
[6] Fer (F.), Lapostolle (P.), Bieth (C.) et CabresPINE (A.), C.E.R.N., MSC 018/999.

[7] Bietih (C.), Cabrespine (A.) et Golditein (Ch.), LA-3609, 1966.

[8] BOUSSARD (D.), LA-3609, 1966.

[9] GoldDSTEIN (Ch.) et LAISNÉ (A.), Nucl. Instr. Methods, 1968, 61, 221. 